Homology, Homotopy and Applications, vol.13(2), 2011, pp.175-195

\title{
L-INFINITY MAPS AND TWISTINGS
}

\author{
JOSEPH CHUANG AND ANDREY LAZAREV
}

(communicated by J.P.C. Greenlees)

\begin{abstract}
We give a construction of an $L_{\infty}$ map from any $L_{\infty}$ algebra into its truncated Chevalley-Eilenberg complex as well as its cyclic and $A_{\infty}$ analogues. This map fits with the inclusion into the full Chevalley-Eilenberg complex (or its respective analogues) to form a homotopy fiber sequence of $L_{\infty}$ algebras. Applications to deformation theory and graph homology are given. We employ the machinery of Maurer-Cartan functors in $L_{\infty}$ and $A_{\infty}$ algebras and associated twistings which should be of independent interest.
\end{abstract}

\section{Introduction}

Let $V$ be a Lie algebra and $f: V \rightarrow \operatorname{Hom}(V, V)$ be the map that associates to $v \in V$ the endomorphism $f(v):=\operatorname{ad}(v): x \mapsto[v, x]$ for $x \in V$. Then $f$ is clearly a Lie algebra homomorphism $V \rightarrow \operatorname{Der}(V, V)$ from $V$ into the Lie algebra of derivations of $V$. If instead $V$ is an associative algebra, $f$ is still a Lie algebra map from the commutator Lie algebra of $V$ into the Lie algebra of derivations of $V$. Furthermore, a variant of this construction works when $V$ has an invariant scalar product $\langle$,$\rangle , so that$ $\langle[a, b], c\rangle=\langle a,[b, c]\rangle$ in the Lie case and $\langle a b, c\rangle=\langle a, b c\rangle$ in the associative case; here $a, b, c \in V$. In this case the image of $f$ lands in the subspace of $\operatorname{Der}(V, V)$ consisting of skew self-adjoint endomorphisms of $V$.

One of the purposes of this paper is to generalize this simple and well-known construction to $L_{\infty^{-}}$and $A_{\infty}$-algebras. This generalization will be applied to characteristic classes of $A_{\infty}$-algebras in a future paper, but we believe that it is of independent interest. A homotopy-invariant analogue of $\operatorname{Der}(V, V)$ for an $L_{\infty}$-algebra $V$ is a truncated Chevalley-Eilenberg complex $\overline{\mathrm{CE}}(V, V)$ which is part of the following homotopy fiber sequence of complexes:

$$
V \stackrel{f}{\longrightarrow} \overline{\mathrm{CE}}(V, V) \stackrel{g}{\longrightarrow} \mathrm{CE}(V, V),
$$

where $\mathrm{CE}(V, V)$ is the usual (untruncated) Chevalley-Eilenberg complex and $f$ is a certain generalization of the map $f=$ ad defined above. Similarly, in the $A_{\infty}$ algebra

The second author is partially supported by an EPSRC research grant.

Received April 12, 2010, revised June 1, 2011; published on November 4, 2011.

2000 Mathematics Subject Classification: 18D50, 57T30, 81T18, $16 \mathrm{E} 45$.

Key words and phrases: differential graded Lie algebra, Maurer-Cartan element, A-infinity algebra, graph homology, Morita equivalence.

Article available at http://intlpress.com/HHA/v13/n2/a12 and doi:10.4310/HHA.2011.v13.n2.a12

Copyright (C) 2011, International Press. Permission to copy for private use granted. 
context, a homotopy-invariant analogue of $\operatorname{Der}(V, V)$ is a truncated Hochschild complex $\operatorname{Hoch}(V, V)$ which is part of the following homotopy fiber sequence of complexes:

$$
V \stackrel{f}{\longrightarrow} \overline{\operatorname{Hoch}}(V, V) \stackrel{g}{\longrightarrow} \operatorname{Hoch}(V, V)
$$

where $\operatorname{Hoch}(V, V)$ is the usual (untruncated) Hochschild complex. Furthermore, in the presence of an invariant scalar product (which gives rise to a cyclic, or symplectic, $A_{\infty}$ - or $L_{\infty}$-algebra $V$, cf. [7]) we have the following homotopy fiber sequences:

$$
V \stackrel{f}{\longrightarrow} \overline{\mathrm{CE}}(V) \stackrel{g}{\longrightarrow} \mathrm{CE}(V),
$$

in the $L_{\infty}$-case; here $\mathrm{CE}(V)$ is the usual (untruncated) Chevalley-Eilenberg complex with trivial coefficients and $\overline{\mathrm{CE}}(V)$ is its truncated version and

$$
V \stackrel{f}{\longrightarrow} \overline{\mathrm{CHoch}}(V, V) \stackrel{g}{\longrightarrow} \mathrm{CHoch}(V, V),
$$

in the $A_{\infty}$-case; here $\mathrm{CHoch}(V)$ is the usual (untruncated) cyclic Hochschild complex and $\overline{\mathrm{CHoch}}(V)$ is its truncated version.

We show that in all four cases the map $f$ can be lifted to an $L_{\infty}$-map $\mathbf{f}$ possessing a certain universal property. Roughly, it says that $\mathbf{f}$ takes any Maurer-Cartan element $\xi$ in $V$ to the Maurer-Cartan element in $\overline{\mathrm{CE}}(V, V)$, (or $\overline{\operatorname{Hoch}}(V, V), \overline{\mathrm{CE}}(V)$, $\overline{\mathrm{CHoch}}(V, V))$ that corresponds to the $L_{\infty}$-structure on $V$ obtained by twisting with $\xi$. The notions of $\infty$-Maurer-Cartan elements and twisted $\infty$-structures are well-known and appear in many papers on homological algebra, rational homotopy theory, mirror symmetry and deformation theory, cf. for example $[\mathbf{8}, \mathbf{1 0}, \mathbf{3}, \mathbf{4}]$. It seems hard to find a paper collecting all necessary facts about $\infty$-Maurer-Cartan elements and twistings and so we give all definitions and proofs of the results that we need. We do, however, make use of the technology of formal noncommutative (symplectic) geometry devised by Kontsevich $[\mathbf{9}]$ and treated at length in $[\mathbf{7}]$ in the context of infinity structures.

The results obtained are used to produce a map from the Chevalley-Eilenberg homology of a cyclic $L_{\infty}$ - or $A_{\infty}$-algebra into the corresponding version of a graph complex.

Finally, we show that the sequences (1), (2), (3), (4) are homotopy fiber sequences of $L_{\infty}$-algebras (as opposed to simply complexes); this makes a connection to a recent work of Fiorenza and Manetti [2], as well as an earlier work by Voronov on derived brackets $[14,15]$.

The paper is organized as follows. The remaining part of the introduction reviews some results and terminology from $L_{\infty^{-}}$and $A_{\infty}$-algebras and their cohomology theories. Section 2 introduces the notion of Maurer-Cartan space in the infinity-context and establishes some basic properties needed later on; these results are undoubtedly known to experts but difficult to find in the literature. The construction of the $L_{\infty^{-}}$ map $\mathbf{f}$ and a relation to the graph homology are given in Section 3 and the last section establishes a connection to the Fiorenza-Manetti construction and applications to deformation theory. 


\section{Acknowledgements}

The authors wish to thank T. Schedler, for useful comments on a preliminary version of this paper, and the referee, for many helpful corrections and suggestions.

\subsection{Notation and conventions}

In this paper we work in the category of $\mathbb{Z} / 2$-graded vector spaces (also known as super-vector spaces) over a field $\mathbf{k}$ of characteristic zero. However all our results (with obvious modifications) continue to hold in the $\mathbb{Z}$-graded context. The adjective 'differential graded' will mean 'differential $\mathbb{Z} / 2$-graded' and will be abbreviated as 'dg'. A (commutative) differential graded (Lie) algebra will be abbreviated as (c)dg(l)a. We will often invoke the notion of a formal ( $\mathrm{dg}$ ) vector space; this is just an inverse limit of finite-dimensional vector spaces. An example of a formal space is $V^{*}$, the k-linear dual to a discrete vector space $V$. A formal vector space comes equipped with a topology and whenever we deal with a formal vector space all linear maps from or into it will be assumed to be continuous; thus we will always have $V^{* *} \cong V$. All of our unmarked tensors are understood to be taken over $\mathbf{k}$. If $V$ is a discrete space and $W=\lim _{\leftarrow} W_{i}$ is a formal space we will write $V \otimes W$ for $\lim _{\leftarrow} V \otimes W_{i}$; thus for two discrete spaces $V$ and $U$ we have $\operatorname{Hom}(V, U) \cong U \otimes V^{*}$. For a $\mathbb{Z} / 2$-graded vector space $V=V_{0} \oplus V_{1}$ the symbol $\Pi V$ will denote the parity reversion of $V$; thus $(\Pi V)_{0}=V_{1}$ while $(\Pi V)_{1}=V_{0}$. The symbol $S_{n}$ stands for the symmetric group on $n$ letters; it is understood to act on the $n$th tensor power of a $\mathbb{Z} / 2$-graded vector space $V^{\otimes n}$ by permuting the tensor factors.

\subsection{Recollections on $L_{\infty}$ - and $A_{\infty}$-algebras}

Our basic reference on $L_{\infty}$ - and $A_{\infty}$-algebras and their cohomology is [7]; we now recall some of the results and terminology from that paper. A non-unital formal (c)dga is an inverse limit of finite-dimensional nilpotent non-unital (c)dgas. We will, however, always work with unital cdgas, and a formal cdga will then mean a cdga obtained by adjoining a unit to a formal non-unital cdga; thus our formal cdgas will be augmented. The augmentation ideal of an augmented cdga $A$ will be denoted by $A_{+}$. There is an unfortunate clash of terminology: our notion of formality is different from the corresponding notion in rational homotopy theory. The main examples of formal dgas and cdgas will be completed tensor and symmetric algebras on (possibly formal) graded vector spaces $V$; these will be denoted by $\hat{T} V$ and $\hat{S} V$ respectively.

Morphisms between and derivations of formal algebras will not be required to be compatible with augmentations. Note however that the prototypical examples above (and indeed most of the examples of formal algebras we consider) are local, and hence continuous morphisms between them automatically preserve augmentations.

An $A_{\infty}$-algebra structure on a graded vector space $V$ is a continuous odd derivation $m$ of the formal dga $\hat{T} \Pi V^{*}$ and an $L_{\infty}$-algebra structure on $V$ is a continuous odd derivation $m$ of the formal cdga $\hat{S} \Pi V^{*}$; additionally $m$ is required to square to zero and have no constant term. The components $m_{i}: T^{i} \Pi V \rightarrow \Pi V$ or $S^{i} \Pi V \rightarrow \Pi V$ of the dual of the restriction of $m$ to $\Pi V^{*}$ are the structure maps of the corresponding

$A_{\infty^{-}}$or $L_{\infty^{-}}$-structure. If $(V, m)$ and $\left(V^{\prime}, m\right)$ are two $A_{\infty^{-}}$or $L_{\infty^{-}}$-algebras then a morphism between them is a continuous (c)dga map $\left(\hat{T} \Pi V^{*}, m^{\prime}\right) \rightarrow\left(\hat{T} \Pi V^{*}, m\right)$ or $\left(\hat{S} \Pi V^{*}, m^{\prime}\right) \rightarrow\left(\hat{S} \Pi V^{*}, m\right)$, respectively. 
The Hochschild complex of an $A_{\infty}$-algebra $(V, m)$ is the dg vector space

$$
\operatorname{Hoch}(V, V):=\left(\operatorname{Der}\left(\hat{T} \Pi V^{*}\right),[-, m]\right)
$$

consisting of (continuous) derivations of $\hat{T} \Pi V^{*}$ with differential $d(x)=[x, m]$ for $x \in \hat{T} \Pi V^{*}$; this is clearly a dgla. The truncated Hochschild complex $\overline{\operatorname{Hoch}}(V, V)$ is the sub-dgla of $\operatorname{Hoch}(V, V)$ consisting of derivations with vanishing constant term.

Similarly the Chevalley-Eilenberg complex $\mathrm{CE}(V, V)$ of an $L_{\infty}$-algebra $(V, m)$ is the dg vector space (Der $\left.\left(\hat{S} \Pi V^{*}\right),[, m]\right)$ consisting of (continuous) derivations of $\hat{S} \Pi V$ supplied with the differential $d(x)=[x, m]$ for $x \in \hat{S} \Pi V^{*}$; this is clearly a dgla. The truncated Chevalley-Eilenberg complex $\overline{\mathrm{CE}}(V, V)$ is the sub-dgla of $\mathrm{CE}(V, V)$ consisting of derivations with vanishing constant term.

We stress that the algebras $\operatorname{Der}\left(\hat{T} \Pi V^{*}\right)$ and $\operatorname{Der}\left(\hat{S} \Pi V^{*}\right)$ of derivations depend only on the dg vector space $V$. On the other hand the complexes $\operatorname{Hoch}(V, V)$ and $\mathrm{CE}(V, V)$ depend on $(V, m)$, the dg vector space $V$ equipped with an $A_{\infty^{-}}$or $L_{\infty^{-}}$ algebra structure.

We note here that it is more traditional to use the terms Hochschild complex and Chevalley-Eilenberg complex of $(V, m)$ to refer to the dg vector spaces $\Pi \operatorname{Hoch}(V, V)$ and $\Pi \mathrm{CE}(V, V)$; this has the unpleasant effect that the dgla structures on these spaces become odd which also introduces additional signs in various formulas, and this is the reason for our convention; a similar remark also applies to the cyclic complexes below.

For a usual associative algebra the complex $\operatorname{Hoch}(V, V)$ has the familiar form

$$
V \rightarrow \operatorname{Hom}(V, V) \rightarrow \cdots \rightarrow \operatorname{Hom}\left(V^{\otimes n}, V\right) \rightarrow \cdots,
$$

whereas the complex $\overline{\operatorname{Hoch}}(V, V)$ has the same Hochschild differential but starts with the term $\operatorname{Hom}(V, V)$. Similarly, the complex $\operatorname{CE}(V, V)$ for a Lie algebra $V$ has the form

$$
V \rightarrow \operatorname{Hom}(V, V) \rightarrow \cdots \rightarrow \operatorname{Hom}\left(\Lambda^{n}(V), V\right) \rightarrow \cdots,
$$

whereas the complex $\overline{\mathrm{CE}}(V, V)$ has the same Chevalley-Eilenberg differential but starts with the term $\operatorname{Hom}(V, V)$. It is clear that, generally, an element in $\overline{\operatorname{Hoch}}(V, V)$ is a (possibly infinite) linear combination of Hochschild cochains, i.e., multilinear maps $(\Pi V)^{\otimes n} \rightarrow \Pi V$, and similarly an element in $\overline{\mathrm{CE}}(V, V)$ is a (possibly infinite) linear combination of Chevalley-Eilenberg cochains, i.e., graded-symmetric multilinear maps $(\Pi V)^{\otimes n} \rightarrow \Pi V$.

In the presence of a graded symmetric, non-degenerate inner product $\langle$,$\rangle on V$ we can define cyclic (also called symplectic) $L_{\infty}$ - and $A_{\infty}$-algebras by the requirement that the expressions $\left\langle m_{i}\left(x_{1}, \cdots, x_{i}\right), x_{i+1}\right\rangle$ be invariant with respect to cyclic permutations of the elements $x_{1}, \cdots, x_{i+1} \in \Pi V$. The corresponding complexes are denoted by $\operatorname{CHoch}(V, V), \overline{\mathrm{CHoch}}(V, V), \mathrm{CE}(V)$ and $\overline{\mathrm{CE}}(V)$ respectively.

So, $\operatorname{CHoch}(V, V)$ is the sub dg subspace of $\operatorname{Hoch}(V, V)$ consisting of cyclically invariant Hochschild cochains, and $\overline{\mathrm{CHoch}}(V, V)$ is the corresponding sub dg vector space of $\overline{\mathrm{CHoch}}(V, V)$. Likewise, $\mathrm{CE}(V)$ is the sub dg subspace of $\mathrm{CE}(V, V)$ consisting of cyclically invariant Chevalley-Eilenberg cochains, and $\overline{\mathrm{CE}}(V)$ is the corresponding sub $\mathrm{dg}$ vector space of $\overline{\mathrm{CE}}(V, V)$.

Note that $\mathrm{CE}(V)$ admits an alternative interpretation as follows. Consider the maximal ideal $\hat{S} \Pi V_{+}^{*} \subset \hat{S} \Pi V^{*}$ consisting of formal power series with vanishing constant 
term. Then the derivation $m$ restricts to $\hat{S} \Pi V_{+}^{*}$ and the dg vector space $\left(\hat{S} \Pi V_{+}^{*}, m\right)$ is isomorphic to $\mathrm{CE}(V)$; this is a consequence of the Poincaré lemma in formal symplectic geometry. Note also that $\mathrm{CE}(V)$ is essentially (disregarding the shift and the lowest degree term) the Chevalley-Eilenberg complex of $(V, m)$ with trivial coefficients.

We will need to consider infinity-algebra structures over a formal cdga $A$. We define an $A$-linear $A_{\infty}$-algebra structure on a graded vector space $V$ to be a continuous odd $A$-linear derivation $m$ of the formal dga $A \otimes \hat{T} \Pi V^{*}$; in addition $m+d_{A} \otimes$ id is required to square to zero and have vanishing constant term. The components $m_{i}: A \otimes T^{i} \Pi V \rightarrow A \otimes \Pi V$ of the $A$-linear dual of the restriction of $m$ to $A \otimes \Pi V^{*}$ are the structure maps of the corresponding $A_{\infty^{-}}$or $L_{\infty}$-structure; they may be viewed as $A$-multilinear maps on $A \otimes \Pi V$. Note that a (k-linear) $A_{\infty}$-algebra $(V, m)$ is also an $A$-linear $A_{\infty}$-algebra $\left(V, m^{A}\right)$, for any formal cdga $A$, by extension of scalars: $m^{A}:=\operatorname{id}_{A} \otimes m$ (equivalently $m_{i}^{A}:=\mathrm{id}_{A} \otimes m_{i}$ ). In a similar way we can introduce the notion of an $A$-linear $L_{\infty}$-algebra structure on a graded vector space $V$. If, in addition, $V$ possesses a graded symmetric, non-degenerate inner product, $A$-linear cyclic $A_{\infty^{-}}$ and $L_{\infty}$-algebra structures on $V$ are defined in an obvious way.

\section{Maurer-Cartan functor and twisting in $L_{\infty}$ and $A_{\infty}$-algebras}

In this section we describe the Maurer-Cartan functor for $L_{\infty^{-}}$and $A_{\infty}$-algebras in both cyclic and non-cyclic cases and associated twisted structures.

Definition 2.1. Let $V$ be a graded vector space.

1. Let $m$ be an $L_{\infty}$-structure on $V$ and $A$ be a formal cdga with maximal ideal $A_{+}$. Then an even element $\xi \in A_{+} \otimes \Pi V$ is Maurer-Cartan if

$$
\left(d_{A} \otimes \mathrm{id}\right)(\xi)+\sum_{i=1}^{\infty} \frac{1}{i !} m_{i}^{A}\left(\xi^{\otimes i}\right)=0 .
$$

2. Let $m$ be an $A_{\infty}$-structure on $V$ and $A$ be a formal dga with maximal ideal $A_{+}$. Then an even element $\xi \in A_{+} \otimes \Pi V$ is Maurer-Cartan if

$$
\left(d_{A} \otimes \mathrm{id}\right)(\xi)+\sum_{i=1}^{\infty} m_{i}^{A}\left(\xi^{\otimes i}\right)=0 .
$$

The set of Maurer-Cartan elements in $A_{+} \otimes \Pi V$ will be denoted by $\operatorname{MC}(V, A)$. The correspondence $(V, A) \mapsto \mathrm{MC}(V, A)$ is functorial in $A$.

\section{Proposition 2.2.}

1. Let $(V, m)$ be an $L_{\infty}$-algebra, and let $A$ be a formal cdga. Then the functor $A \mapsto \mathrm{MC}(V, A)$ is represented by the formal cdga $\left(\hat{S} \Pi V^{*}, m\right)$.

2. Let $(V, m)$ be an $A_{\infty}$-algebra, and let $A$ be a formal dga. Then the functor $A \mapsto \operatorname{MC}(V, A)$ is represented by the formal cdga $\left(\hat{T} \Pi V^{*}, m\right)$.

Proof. For (1) let $f: \hat{S} \Pi V^{*} \rightarrow A$ be a map of formal cdgas. Forgetting the differentials, such a map is equivalent to a continuous linear map $\Pi V^{*} \rightarrow A$, which in turn 
determines an element $\xi_{f} \in A \otimes \Pi V$. The condition that $f$ commutes with differentials could be rewritten as the commutative diagram:

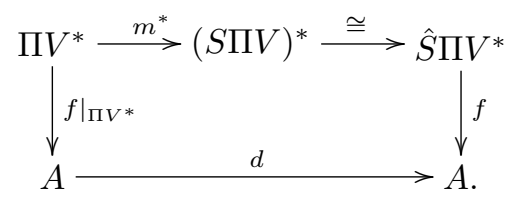

Note that the canonical isomorphism

$$
\left(S^{n} \Pi V\right)^{*}:=\left(\left(T^{n} \Pi V\right)_{S_{n}}\right)^{*}=\left(\hat{T}^{n} \Pi V^{*}\right)^{S_{n}} \rightarrow\left(\hat{T}^{n} \Pi V^{*}\right)_{S_{n}}=: S^{n} \Pi V^{*}
$$

identifying invariants with coinvariants has the form

$$
\left(\hat{T}^{n} \Pi V^{*}\right)^{S_{n}} \ni x_{1} \otimes \cdots \otimes x_{n} \mapsto \frac{1}{n !} x_{1} \otimes \cdots \otimes x_{n} \in\left(\hat{T}^{n} \Pi V^{*}\right)_{S_{n}} .
$$

Taking this into account we obtain that the commutativity of the diagram (5) is equivalent to the Maurer-Cartan equation for $\xi_{f}$ :

$$
\left(d_{A} \otimes \mathrm{id}\right)\left(\xi_{f}\right)+m_{1}^{A}\left(\xi_{f}\right)+\frac{1}{2 !} m_{2}^{A}\left(\xi_{f} \otimes \xi_{f}\right)+\cdots=0,
$$

where $m_{i}^{A}:(A \otimes \Pi V)^{\otimes i} \rightarrow A \otimes \Pi V$ is the $i$ th higher bracket in the $A$-linear $L_{\infty^{-}}$ algebra $V$.

We omit the proof of (2) which is similar to, but simpler than, that of (1) in that one does not to have to worry about identification of $S_{n}$-invariants with $S_{n}$-coinvariants (and thus, no factorials will appear in the formulas).

Remark 2.3. There is another version of the MC-functor which is occasionally useful. For an $L_{\infty}$-algebra $(V, m)$ it associates to a not necessarily formal cdga $A$ the set $\xi \in$ $\Pi V \otimes A$ such that $\sum_{i=1}^{\infty} \frac{1}{i !} m_{i}^{A}\left(\xi^{\otimes i}\right)=0$ and similarly when $(V, m)$ is an $A_{\infty}$-algebra. The formal series above does not have to converge and to justify this definition one has to impose some additional restrictions on $V$, e.g., that it possesses only finitely many non-zero higher products as is the case when $V$ is a dga or a dgla. In this situation (at least when $V$ is finite-dimensional) the functor $\operatorname{MC}(V,-)$ is still represented by a cdga $\left(S \Pi V^{*}, m\right)$ in the $L_{\infty}$-case and $\left(T \Pi V^{*}, m\right)$ in the $A_{\infty}$-case (notice the absence of the completion). All statements in this section have obvious analogues in this context.

Remark 2.4. Let $(V, m)$ be an $A_{\infty}$-algebra. Viewing $m$ as a map $\Pi V^{*} \rightarrow \hat{T} \Pi V^{*}$ and composing with the projection $\hat{T} \Pi V^{*} \rightarrow \hat{S} \Pi V^{*}$ we obtain a map $\bar{m}: \Pi V^{*} \rightarrow \hat{S} \Pi V^{*}$ which, obviously, gives an $L_{\infty}$-structure on $V$ (this is an infinity version of the familiar commutator Lie algebra construction). Furthermore, for a formal cgda $A$ the MaurerCartan equations in $A \otimes \Pi V$ are the same regardless of whether $(V, m)$ is considered as an $L_{\infty^{-}}$or $A_{\infty}$-algebra; in other words an element $\xi \in A \otimes \Pi V$ is an $L_{\infty}$-MCelement if and only if it is an $A_{\infty}$-MC-element.

The Yoneda lemma now gives:

\section{Corollary 2.5.}

1. Let $(V, m)$ and $\left(V^{\prime}, m^{\prime}\right)$ be two $L_{\infty}$-structures and $A$ be a formal cdga. Then any $L_{\infty}$-map $V \rightarrow V^{\prime}$ determines and is determined by a morphism of functors (in the variable $A): \operatorname{MC}(V, A) \rightarrow \operatorname{MC}\left(V^{\prime}, A\right)$. 
2. Let $(V, m)$ and $\left(V^{\prime}, m^{\prime}\right)$ be two $A_{\infty}$-structures and $A$ be a formal dga. Then any $A_{\infty}-$ map $V \rightarrow V^{\prime}$ determines and is determined by a morphism of functors (in the variable $A): \operatorname{MC}(V, A) \rightarrow \operatorname{MC}\left(V^{\prime}, A\right)$.

Proof. We only have to note that an $L_{\infty}$-map $V \rightarrow V^{\prime}$ is by definition a (continuous) map of formal cdgas $\left(\hat{S} \Pi V^{*}, m^{\prime}\right) \rightarrow\left(\hat{S} \Pi V^{*}, m\right)$ and an $A_{\infty}$-map $V \rightarrow V^{\prime}$ is a (continuous) map of formal dgas $\left(\hat{T} \Pi V^{\prime *}, m^{\prime}\right) \rightarrow\left(\hat{T} \Pi V^{*}, m\right)$.

We will now turn to twistings. Let $(V, m)$ be an $L_{\infty}$-algebra and $A$ be a formal cdga. We shall explain how a Maurer-Cartan element $\xi \in \mathrm{MC}(V, A)$ gives rise to a new $L_{\infty}$-algebra $\left(V, m^{\xi}\right)$ on the same underlying space; the derivation $m^{\xi}$ is obtained by applying an appropriate automorphism of $\operatorname{Der}(\hat{T} \Pi V)$ to $m$, or equivalently as a constant shift by $\xi$ in the arguments of $m$. Both points of view are useful and so we describe both in detail and explain the relationship between them.

We may regard $\xi$ as a (formal) $A$-linear derivation of the formal algebra $A \otimes \hat{S} \Pi V^{*}$; indeed $\xi$ could be viewed as a linear function on $\Pi V^{*} \cong 1 \otimes \Pi\left(V^{*}\right) \subset A \otimes \hat{S} \Pi V^{*}$ and we extend it to the whole of $A \otimes \hat{S} \Pi V^{*}$ by the $A$-linear Leibniz rule.

Consider now $e^{\xi}:=\mathrm{id}+\xi+\cdots$ as an automorphism of $A \otimes \hat{S} \Pi V^{*}$; it is clear that this formal power series converges. This automorphism acts by conjugations on derivations of $A \otimes \hat{S} \Pi V^{*}$; in particular on those of square zero; moreover it turns out that it takes one $A$-linear $L_{\infty}$-structure on $V$ to another such structure; the precise statement is given below.

A similar discussion is applicable to $A_{\infty}$-algebras as well. Namely, let $(V, m)$ be an $A_{\infty}$-algebra, $A$ be a formal dga and $\xi \in \mathrm{MC}(V, A)$. We shall view $\xi$ as a (formal) $A$-linear derivation of the formal algebra $A \otimes \hat{T} \Pi V^{*}$; indeed $\xi$ could be viewed as a linear function on $\Pi V^{*} \cong 1 \otimes \Pi\left(V^{*}\right) \subset A \otimes \hat{T} \Pi V^{*}$ and we extend it to the whole of $A \otimes \hat{T} \Pi V^{*}$ by the $A$-linear Leibniz rule. Then the automorphism $e^{\xi}$ acts on the set of $A$-linear $A_{\infty}$-structures on $V$. Here is the general formulation:

\section{Theorem 2.6.}

1. (a) Let $(V, m)$ be an $L_{\infty}$-algebra, $A$ be a formal cdga and $\xi \in A_{+} \otimes \Pi V$. Then $m^{\xi}:=e^{\xi} m^{A} e^{-\xi}$, considered as a derivation of $A \otimes \hat{S} \Pi V^{*}$, has no constant term (and thus, gives another $A$-linear $L_{\infty}$-structure on $V$ ) if and only if $\xi$ is Maurer-Cartan. The process of passing from $m$ to $m^{\xi}$ is called twisting by a Maurer-Cartan element $\xi$.

(b) For $x_{1}, \cdots, x_{n} \in \Pi V$ the following formula holds:

$$
m_{n}^{\xi}\left(x_{1}, \cdots, x_{n}\right)=\sum_{i=0}^{\infty} \frac{1}{i !} m_{n+i}^{A}\left(\xi, \cdots, \xi, x_{1}, \cdots, x_{n}\right) .
$$

2. (a) Let $(V, m)$ be an $A_{\infty}$-algebra, $A$ be a formal dga and $\xi \in A_{+} \otimes \Pi V$. Then $m^{\xi}:=e^{\xi} m^{A} e^{-\xi}$, considered as a derivation of $A \otimes \hat{T} \Pi V^{*}$, has no constant term (and thus, gives another A-linear $A_{\infty}$-structure on $V$ ) if and only if $\xi$ is Maurer-Cartan. The process of passing from $m$ to $m^{\xi}$ is called twisting by a Maurer-Cartan element $\xi$. 
(b) For $x_{1}, \cdots, x_{n} \in \Pi V$ the following formula holds:

$$
m_{n}^{\xi}\left(x_{1}, \cdots, x_{n}\right)=\sum_{i=0}^{\infty} m_{n+i}^{A}\left(\xi, \cdots, \xi \mid x_{1}, \cdots, x_{n}\right),
$$

where

$$
m_{n+i}^{A}\left(\xi, \cdots, \xi \mid x_{1}, \cdots, x_{n}\right):=\sum m_{n+i}^{A}\left(z_{1}, \cdots, z_{n+i}\right),
$$

the sum running over all $\left(\begin{array}{c}n+i \\ n\end{array}\right)$ sequences $z_{1}, \cdots, z_{n+i}$ containing $x_{1}, \cdots, x_{n}$ in order, together with $i$ copies of $\xi$.

Proof. We will restrict ourselves to proving part (1), the proof for (2) is similar but simpler. Choose a basis $x_{i}$ in $V$ and the corresponding linear coordinates $x^{i}$ in $\Pi V^{*}$. Then we can write $\xi=\sum_{i} \xi^{i} x_{i}$ where $\xi^{i} \in A$, and any element in $A \otimes \hat{S} \Pi V^{*}$ can be written as a formal power series $f\left(x^{1}, \cdots, x^{n}, \cdots,\right)$ with coefficients in $A$. The action of $e^{\xi}$ on $f$ is then given as $\xi \cdot f=f\left(x^{1}+\xi^{1}, \cdots, x^{n}+\xi^{n}, \cdots\right)$.

Furthermore, for a derivation $\eta:=\sum f^{i}\left(x^{1}, \cdots, x^{n}, \cdots,\right) \partial_{x^{i}}$ we have

$$
e^{\xi} \eta e^{-\xi}=\sum_{i} f^{i}\left(x^{1}+\xi^{1}, \cdots, x^{n}+\xi^{n}, \cdots\right) \partial_{x^{i}} .
$$

Let us now take $\eta=m^{A}$, the $A$-linear derivation of $A \otimes \hat{S} \Pi V^{*}$ corresponding to a given $L_{\infty}$-structure on $V$. We see that the derivation $e^{\xi} \eta e^{-\xi}$ has no constant term (and thus, determines another $L_{\infty}$-structure) if and only if $\sum_{i} f^{i}\left(\xi^{1}, \cdots, \xi^{n}, \cdots\right)=0$.

The right hand side of (8) is a sum of monomials of the form

$$
f_{i_{1} \cdots i_{n}}\left(x^{i_{1}}+\xi^{i_{1}}\right)\left(x^{i_{2}}+\xi^{i_{1}}\right) \cdots\left(x^{i_{n}}+\xi^{i_{n}}\right) \partial_{x_{i}},
$$

where $f_{i_{1}, \cdots, i_{n}} \in A$. Every such monomial represents a linear map $\Pi V^{*} \rightarrow A \otimes \hat{S} \Pi V^{*}$ and the components of the dual map $S^{n} \Pi V \rightarrow A \otimes \Pi V$ correspond to the matrix elements of $m^{\xi}$. It follows that equation (6) holds; moreover for $n=0$ the constant part of the derivation $m^{\xi}$ corresponds to $m_{0}:=\sum_{i=0}^{\infty} \frac{1}{i !} m_{i}(\xi, \cdots, \xi)$ and so its vanishing is indeed equivalent to the Maurer-Cartan condition as claimed.

Example 2.7. One of the most important special cases of the construction above concerns the case when $V$ is a dgla or dga (as opposed to a general $L_{\infty^{-}}$or $A_{\infty^{-}}$-algebra). In that situation the tensoring with a formal cdga $A$ is unnecessary and we can consider $\operatorname{MC}(V):=\left\{\xi \in \Pi V: d \xi+\frac{1}{2}[\xi, \xi]=0\right\}$ (note that this equation is the same in the associative situation since then $\frac{1}{2}[\xi, \xi]=\xi^{2}$ ) In this situation it is easy to check that the corresponding twisted dga or dgla will have the same product as $V$ and the twisted differential: $d^{\xi}(x)=d x+[\xi, x]$ for $x \in V$.

Remark 2.8. Note that the formulas (6) and (7) do not contain signs; this happens because our $m_{i}$ 's are viewed as multi-linear maps on $\Pi V$; accordingly $\xi$ is treated as an even element (or constant derivation) and so permuting it past other element does not give rise to signs.

We have the following easy result; in order to formulate it we observe that the definition of $\operatorname{MC}(V, A)$ extends in an obvious way to any $A$-linear $L_{\infty}$ - or $A_{\infty}$-algebra $V$. Here and sometimes later on we will use the shorthand $V^{\xi}\left(\right.$ or $\left.(A \otimes V)^{\xi}\right)$ to denote the $A$-linear $L_{\infty}$ - or $A_{\infty}$-algebra obtained by twisting by a Maurer-Cartan element $\xi$. 


\section{Proposition 2.9.}

1. Let $V$ be an $L_{\infty}$-algebra, $A$ be a formal cdga and $\xi \in \mathrm{MC}(V, A)$. Then for $\eta \in$ $\mathrm{MC}(V, A)$ we have $\eta-\xi \in \mathrm{MC}\left(V^{\xi}, A\right)$ and there is a one-to-one correspondence $\mathrm{MC}(V, A) \rightarrow \mathrm{MC}\left(V^{\xi}, A\right)$ given by $\eta \mapsto \eta-\xi$.

2. Let $V$ be an $A_{\infty}$-algebra, $A$ be a formal dga and $\xi \in \operatorname{MC}(V, A)$. Then for $\eta \in$ $\operatorname{MC}(V, A)$ we have $\eta-\xi \in \mathrm{MC}\left(V^{\xi}, A\right)$ and there is a one-to-one correspondence $\mathrm{MC}(V, A) \rightarrow \mathrm{MC}\left(V^{\xi}, A\right)$ given by $\eta \mapsto \eta-\xi$.

Proof. We will only give a proof for (1) since it will also work for (2) without any changes. Denote by $m$ the given $L_{\infty}$-structure on $V$. If $\eta \in \mathrm{MC}(V, A)$ then

$$
e^{\eta-\xi} m^{\xi} e^{-\eta+\xi}=e^{\eta-\xi} e^{\xi} m e^{-\xi} e^{-\eta+\xi}=e^{\eta} m e^{-\eta} .
$$

Therefore $e^{\eta-\xi} m^{\xi} e^{-\eta+\xi}$ has no constant term and $\eta-\xi \in \mathrm{MC}\left(V^{\xi}, A\right)$. This argument is clearly reversible so $\eta-\xi \in \mathrm{MC}\left(V^{\xi}, A\right)$ if and only if $\eta \in \mathrm{MC}(V, A)$.

Remark 2.10. In the next section we will need the analogue of the above result when $V$ is a dgla and $A$ is a not necessarily formal (see Remark 2.3). The proof will be the same, verbatim.

The twisted infinity structures are compatible with invariant scalar products in the following sense.

\section{Proposition 2.11.}

1. Let $(V, m)$ be a cyclic $L_{\infty}$-algebra, $A$ be a formal cdga and $\xi \in \operatorname{MC}(V, A)$. Then $\left(V, m^{\xi}\right)$ is an A-linear cyclic $L_{\infty}$-algebra.

2. Let $(V, m)$ be a cyclic $A_{\infty}$-algebra, $A$ be a formal dga and $\xi \in \operatorname{MC}(V, A)$. Then $\left(V, m^{\xi}\right)$ is an A-linear cyclic $A_{\infty}$-algebra.

Proof. The proof is the same for both cases; let us therefore consider the $L_{\infty}$-case. Recall that $\hat{S} \Pi(A \otimes V)^{*}$ is a formal $A$-supermanifold with a symplectic structure determined by a given scalar product on $V$ and that $m$ is a symplectic vector field. The adjoint action of the constant vector field $\xi$ is a translation; therefore it preserves symplectic vector fields. It follows that $m^{\xi}$ is symplectic as required.

Alternatively, one can check directly, using formulas (6) that $m^{\xi}$ has the desired cyclic invariance property.

\subsection{Maurer-Cartan elements in Hochschild and Chevalley-Eilenberg complexes}

Let $V$ be a graded vector space and $A$ be a formal cdga. Recall that an $A$-linear $L_{\infty^{-}}$ structure on $V$ is an odd (continuous) $A$-linear derivation $m$ of the formal cdga $A \otimes$ $\hat{S} \Pi V^{*}$ without constant term and of square zero. Put another way, $m \in \operatorname{MC}(\mathfrak{g}, A)$, where $\mathfrak{g}=\operatorname{Der}_{0}\left(\hat{S} \Pi V^{*}\right)$, the graded Lie algebra of continuous derivations of $\hat{S} \Pi V^{*}$ without constant term. Let us consider the corresponding $A$-linear dgla $(A \otimes \mathfrak{g})^{m}$ obtained by twisting the graded Lie algebra $A \otimes \mathfrak{g}$ by $m$; recall that the twisted differential will have the form $d(x)=[m, x]$ for $x \in A \otimes \mathfrak{g}$. By definition, the dgla $(A \otimes \mathfrak{g})^{m}$ is isomorphic to the truncated Chevalley-Eilenberg complex $\overline{\mathrm{CE}}_{A}(V, V)=$ $A \otimes \overline{\mathrm{CE}}(V, V)$ of the $A$-linear $L$-infinity algebra $(V, m)$. 
Similarly, an $A$-linear $A_{\infty}$-algebra structure on $V$ is a Maurer-Cartan element $m$ in the graded Lie algebra $A \otimes \mathfrak{h}$, where $\mathfrak{h}=\operatorname{Der}_{0}\left(\hat{T} \Pi V^{*}\right)$ is the graded Lie algebra of continuous derivations of the formal dga $\hat{T} \Pi V^{*}$ with vanishing constant term; the twisted dgla $(A \otimes \mathfrak{h})^{m}$ is just the truncated Hochschild complex $\overline{\operatorname{Hoch}}_{A}(V, V)=$ $A \otimes \overline{\operatorname{Hoch}}(V, V)$ of $(V, m)$.

These observations have cyclic versions: suppose that $(V, m)$ is an $A$-linear cyclic $L_{\infty}$-algebra. That means that the space $V^{*}$ is supplied with a (graded) symplectic structure and that $m$ is a symplectic derivation. Consider the Lie subalgebra $\tilde{\mathfrak{g}}$ of $\mathfrak{g}$ consisting of symplectic derivations; then $m \in \mathrm{MC}(\tilde{\mathfrak{g}}, A)$ and the twisted dgla $(A \otimes$ $\tilde{\mathfrak{g}})^{m}$ is identified with the truncated cyclic complex $\overline{\mathrm{CE}}_{A}(V)=A \otimes \overline{\mathrm{CE}}(V)$ of $(V, m)$.

Similarly if $(V, m)$ is a $A$-linear cyclic $A_{\infty}$-algebra then the space $V^{*}$ is supplied with a (graded) symplectic structure and $m$ is a (noncommutative) symplectic derivation. Consider the Lie subalgebra $\tilde{\mathfrak{h}}$ of $\mathfrak{h}$ consisting of symplectic derivations; then $m \in \operatorname{MC}(\tilde{\mathfrak{g}}, A)$ and the twisted dgla $(A \otimes \tilde{\mathfrak{g}})^{m}$ is identified with the truncated cyclic complex $\overline{\mathrm{CHoch}}_{A}(V, V)=A \otimes \overline{\mathrm{CHoch}}(V, V)$ of $(V, m)$.

Then we have the following result which is a direct consequence of Proposition 2.9.

Proposition 2.12. Let $A$ be a cdga:

1. (a) Let $(V, m)$ be an $L_{\infty}$-algebra. Then there is a bijection between the set of $A$-linear $L_{\infty}$-structures on $V$ and $\mathrm{MC}(\overline{\mathrm{CE}}(V, V), A)$; namely any A-linear $L_{\infty}$-structure $m^{\prime}$ determines an $M C$-element $m^{\prime}-m \in \mathrm{MC}(\overline{\mathrm{CE}}(V, V), A)$.

(b) Let $(V, m)$ be a cyclic $L_{\infty}$-algebra. Then there is a bijection between the set of $A$-linear cyclic $L_{\infty}$-structures on $V$ and $\mathrm{MC}(\overline{\mathrm{CE}}(V), A)$; any A-linear cyclic $L_{\infty}$-structure $m^{\prime}$ determines an $M C$-element $m^{\prime}-m \in \mathrm{MC}(\overline{\mathrm{CE}}(V), A)$.

2. (a) Let $(V, m)$ be an $A_{\infty}$-algebra. Then there is a bijection between the set of $A$-linear $A_{\infty}$-structures on $V$ and $\operatorname{MC}(\overline{\operatorname{Hoch}}(V, V), A)$; namely any A-linear $A_{\infty}$-structure $m^{\prime}$ determines an $M C$-element

$$
m^{\prime}-m \in \operatorname{MC}(\overline{\operatorname{Hoch}}(V, V), A) .
$$

(b) Let $(V, m)$ be a cyclic $A_{\infty}$-algebra. Then there is a bijection between the set of A-linear cyclic $A_{\infty}$-structures on $V$ and $\mathrm{MC}(\overline{\mathrm{CHoch}}(V, V), A)$; namely any $A$-linear cyclic $A_{\infty}$-structure $m^{\prime}$ determines an $M C$-element $m^{\prime}-m \in$ $\mathrm{MC}(\overline{\mathrm{CHoch}}(V, V), A)$.

Remark 2.13. There is an obvious analogue of the above result in the situation when $A$ is a not necessarily formal cdga, cf. Remark 2.10 .

\section{Main construction}

In this section we describe an $L_{\infty}$-map $\mathbf{f}=\left(f_{1}, f_{2}, \cdots\right)$ from an $L_{\infty}$ - or $A_{\infty}$-algebra to its truncated Chevalley-Eilenberg or Hochschild complex as well as the analogues in the cyclic situation. The first component $f_{1}$ is the map $f$ appearing in the homotopy fiber sequences (1.1)-(1.4). 


\section{Theorem 3.1.}

1. (a) Let $(V, m)$ be an $L_{\infty}$-algebra. Then the maps $f_{r}:(\Pi V)^{\otimes r} \rightarrow \Pi \overline{\mathrm{CE}}(V, V)$ given by the formulas

$f_{r}\left(w_{1}, \cdots, w_{r}\right)\left(x_{1}, \cdots, x_{n}\right)=m_{r+n}\left(w_{1}, \cdots, w_{r}, x_{1}, \cdots, x_{n}\right),\left(w_{i}, x_{i} \in \Pi V\right)$

define an $L_{\infty}$-map $\mathbf{f}: V \rightarrow \overline{\mathrm{CE}}(V, V)$. The map $\mathbf{f}$ is characterized by the property that for any formal algebra $A$ an $M C$-element $\xi \in \mathrm{MC}(V, A)$ is mapped to an $M C$-element in $\mathrm{MC}(\overline{\mathrm{CE}}(V, V), A)$ corresponding to the twisting of $A \otimes V$ by the element $\xi$.

(b) Let $(V, m)$ be a cyclic $L_{\infty}$-algebra. Then the maps $f_{r}:(\Pi V)^{\otimes r} \rightarrow \Pi \overline{\mathrm{CE}}(V)$ are given by the formulas (9) define an $L_{\infty}$-map $\mathbf{f}: V \rightarrow \overline{\mathrm{CE}}(V)$. The map $\mathbf{f}$ is characterized by the property that for any formal algebra $A$ an $M C$ element $\xi \in \mathrm{MC}(V, A)$ is mapped to an MC-element in $\mathrm{MC}(\overline{\mathrm{CE}}(V), A)$ corresponding to the twisting of $A \otimes V$ by the element $\xi$.

2. (a) Let $(V, m)$ be an $A_{\infty}$-algebra. Then the maps $f_{r}:(\Pi V)^{\otimes r} \rightarrow \Pi \overline{\mathrm{Hoch}}(V, V)$ given by the formulas

$$
f_{r}\left(w_{1}, \cdots, w_{r}\right)\left(x_{1}, \cdots, x_{n}\right)=\sum_{\sigma \in S_{r}} \frac{1}{r !} m_{r+n}\left(\sigma\left(w_{1} \otimes \cdots \otimes w_{r}\right) \mid x_{1}, \cdots, x_{n}\right),
$$

define an $L_{\infty}-\operatorname{map} \mathbf{f}: V \rightarrow \overline{\operatorname{Hoch}}(V, V)$. Here $m_{r+n}\left(w_{i_{1}}, \cdots, w_{i_{r}} \mid x_{1}, \cdots, x_{n}\right)$ is defined as $\sum \pm m_{r+n}\left(z_{1}, \cdots, z_{r+n}\right)$, where the sum ranges over all $\left(\begin{array}{c}r+n \\ s\end{array}\right)$ shuffles of the sequences $w_{i_{1}}, \cdots, w_{i_{r}}$ and $x_{1}, \cdots, x_{n}$, and the sign is determined by the Koszul sign rule. The map $\mathbf{f}$ is characterized by the property that for any formal algebra $A$ an $M C$-element $\xi \in \mathrm{MC}(V, A)$ is mapped to an $M C$-element in $\mathrm{MC}(\overline{\operatorname{Hoch}}(V, V), A)$ corresponding to the twisting of $A \otimes V$ by the element $\xi$.

(b) Let $(V, m)$ be a cyclic $A_{\infty}$-algebra. Then $f_{r}:(\Pi V)^{\otimes r} \rightarrow \Pi \overline{\mathrm{CHoch}}(V, V)$ given by the formulas (10) define an $L_{\infty}$-map $\mathbf{f}: V \rightarrow \overline{\mathrm{CHoch}}(V, V)$. The map $\mathbf{f}$ is characterized by the property that for any formal algebra $A$ an $M C$ element $\xi \in \mathrm{MC}(V, A)$ is mapped to an MC-element in $\mathrm{MC}(\overline{\mathrm{CHoch}}(V, V), A)$ corresponding to the twisting of $A \otimes V$ by the element $\xi$.

Remark 3.2. The maps $\mathbf{f}$ in the $L_{\infty}$ - and $A_{\infty}$-contexts are compatible in the following sense.

Let $(V, m)$ be an $A_{\infty}$-algebra; it could be regarded as an $L_{\infty}$-algebra, q.v. Remark 2.4. Furthermore, extending this observation, one sees that there are maps of dglas $\operatorname{Hoch}(V, V) \rightarrow \mathrm{CE}(V, V)$ and $\overline{\operatorname{Hoch}}(V, V) \rightarrow \overline{\mathrm{CE}}(V, V)$. Then straightforward inspection shows that there is a commutative diagram of $L_{\infty}$-algebras and maps:

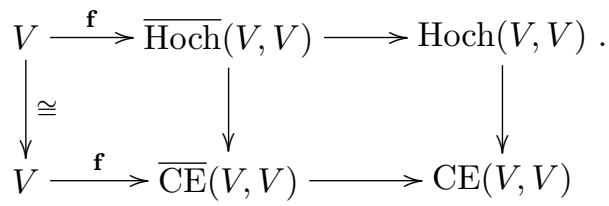

Moreover, a similar statement also holds in the cyclic context. 


\subsection{Proof of Theorem 3.1: $L_{\infty}$-algebra case}

Let $(V, m)$ be an $L_{\infty}$-structure on a graded vector space $V$. To construct an $L_{\infty^{-}}$ map $V \rightarrow \overline{\mathrm{CE}}(V, V)$ it is sufficient to exhibit a map

$$
\mathrm{MC}(V, A) \rightarrow \mathrm{MC}(\overline{\mathrm{CE}}(V, V), A)
$$

for any formal cdga $A$, which is functorial in $A$. Indeed, the functor $A \mapsto \mathrm{MC}(V, A)$ is represented by the cdga $\left(\hat{S} \Pi V^{*}, m\right)$ and thus, by Yoneda's lemma such a functorial map is induced by a cdga map $\hat{S} \Pi \overline{\mathrm{CE}}(V, V)^{*} \rightarrow \hat{S} \Pi V^{*}$ i.e., the desired $L_{\infty}$-map.

Let $\xi \in \mathrm{MC}(V, A)$; it gives rise via twisting to the $A$-linear $L_{\infty}$-algebra $V^{\xi}$ and hence, by Proposition 2.12 (more precisely, its version described in Remark 2.3), to an $\mathrm{MC}$ element $m^{\xi}-m \in \mathrm{MC}(\overline{\mathrm{CE}}(V, V), A)$. Note that while in general neither $m$ nor $m^{\xi}$ is contained in $A_{+} \otimes \Pi \overline{\mathrm{CE}}(V, V)$, their difference is. So the correspondence $\xi \mapsto m^{\xi}-m$ defines the desired map $\mathrm{MC}(V, A) \rightarrow \mathrm{MC}(\overline{\mathrm{CE}}(V, V), A)$.

We will now describe the map $\mathbf{f}$ explicitly. To do this we perform the above argument for the universal example $A=\left(\hat{S} \Pi V^{*}, m\right)$. Consider the canonical MC-element $\xi=\sum w^{i} \otimes w_{i} \in \hat{S} \Pi V^{*} \otimes \Pi V$, where $\left\{w_{i}\right\}$ is a basis of $\Pi V$ and $\left\{w^{i}\right\}$ its dual basis in $\Pi V^{*} \subset \hat{S} \Pi V^{*}$. The corresponding twisted $A$-linear $L_{\infty}$-structure on $A \otimes V$ is given by

$$
\begin{aligned}
m_{n}^{\xi}\left(x_{1}, \cdots, x_{n}\right) & =\sum_{r=0}^{\infty} \frac{1}{r !} m_{r+n}^{\hat{S} \Pi V^{*}}(\underbrace{\xi, \cdots, \xi}_{r}, x_{1}, \cdots, x_{n}) \\
& =\sum_{r=0}^{\infty} \frac{1}{r !} m_{r+n}^{\hat{S} \Pi V^{*}}\left(\sum_{i_{1}} w^{i_{1}} \otimes w_{i_{1}}, \cdots, \sum_{i_{r}} w^{i_{r}} \otimes w_{i_{r}}, x_{1}, \cdots, x_{n}\right) \\
& =\sum_{\substack{r \geqslant 0 \\
i_{1}, \cdots, i_{r}}} \frac{1}{r !} w^{i_{r}} \cdots w^{i_{1}} \otimes m_{r+n}\left(w_{i_{1}}, \cdots, w_{i_{r}}, x_{1}, \cdots, x_{n}\right)
\end{aligned}
$$

for $x_{1}, \cdots, x_{n} \in \Pi V$

This structure is interpreted as an MC-element $\tilde{\xi}=\left(\tilde{\xi}_{n}\right)$ in the dgla

$$
\hat{S} \Pi V^{*} \otimes \overline{\mathrm{CE}}(V, V)=\bigoplus_{n \geqslant 1} \hat{S} \Pi V^{*} \otimes \operatorname{Hom}\left(S^{n} \Pi V, \Pi V\right),
$$

where

$$
\tilde{\xi}_{n}\left(x_{1}, \cdots, x_{n}\right)=\sum_{\substack{r \geqslant 1 \\ i_{1}, \cdots, i_{r}}} \frac{1}{r !} w^{i_{r}} \cdots w^{i_{1}} \otimes m_{r+n}\left(w_{i_{1}}, \cdots, w_{i_{r}}, x_{1}, \cdots, x_{n}\right) .
$$

(Note that the only the $r>0$ terms of the sum (11) contribute here.) Finally, interpreting the MC-element $\tilde{\xi} \in \hat{S} \Pi V^{*} \otimes \overline{\mathrm{CE}}(V, V)$ as an $L_{\infty}$-map $\mathbf{f}=\left(f_{r}\right)$ from $V$ into

$$
\overline{\mathrm{CE}}(V, V)=\bigoplus_{n \geqslant 1} \operatorname{Hom}\left(S^{n} \Pi V, \Pi V\right),
$$

we obtain the desired formula (9). The factorial disappears in (9) for the same reason that it appears in the proof of part (1) of Proposition 2.2.

Now assume $V$ is a cyclic $L_{\infty}$-algebra. Then the above $L_{\infty}$-map $V \rightarrow \overline{\mathrm{CE}}(V, V)$ factors through $\overline{\mathrm{CE}}(V)$. This follows directly from the fact that a Maurer-Cartan twisting of a cyclic $L_{\infty}$-algebra is again cyclic, cf. Proposition 2.11 . 


\subsection{Proof of Theorem 3.1: $A_{\infty}$-algebra case}

Now we turn to the closely related associative case. Let $(V, m)$ be an $A_{\infty}$-algebra. We will construct an $L_{\infty}$-map from the associated $L_{\infty}$-algebra $(V, \bar{m})$ (cf. Remark 2.4) to the dgla $\overline{\operatorname{Hoch}}(V, V)$.

To construct such a map it suffices to exhibit a map

$$
\operatorname{MC}(V, A) \rightarrow \operatorname{MC}(\overline{\operatorname{Hoch}}(V, V), A)
$$

for any formal cdga $A$, which is functorial in $A$. Indeed, the functor $A \mapsto \mathrm{MC}(V, A)$ is represented by the cdga $\left(\hat{S} \Pi V^{*}, \bar{m}\right)$ and thus, by Yoneda's lemma, such a functorial map is induced by a cdga map $\hat{S} \Pi \overline{H o c h}(V, V)^{*} \rightarrow \hat{S} \Pi V^{*}$ i.e., the desired $L_{\infty}$-map.

Let $\xi \in \mathrm{MC}(V, A)$; it gives rise to a twisted $A$-linear $A_{\infty}$-algebra $(A \otimes V)_{\xi}$. Hence it determines the MC-element $m^{\xi}-m$ in the dgla $A \otimes \overline{\operatorname{Hoch}}(V, V)$. So the correspondence $\xi \mapsto m^{\xi}-m$ defines the desired map $\operatorname{MC}(V, A) \rightarrow \operatorname{MC}(\overline{\operatorname{Hoch}}(V, V), A)$.

We will now describe the map $\mathbf{f}:=\left(f_{1}, f_{2}, \cdots\right)$ explicitly. Consider the canonical MC-element $\xi=\sum w^{i} \otimes w_{i} \in \hat{S} \Pi V^{*} \otimes \Pi V$, where $\left\{w_{i}\right\}$ is a basis of $\Pi V$ and $\left\{w^{i}\right\}$ its dual basis in $\Pi V^{*} \subset \hat{S} \Pi V^{*}$. The corresponding twisted $A$-linear $A_{\infty}$-structure on $\hat{S} \Pi V^{*} \otimes V$ is given by the product

$$
\begin{aligned}
m_{n}^{\xi}\left(x_{1}, \cdots, x_{n}\right) & =\sum_{r=0}^{\infty} m_{r+n}^{\hat{S} \Pi V^{*}}(\underbrace{\xi, \cdots, \xi}_{r} \mid x_{1}, \cdots, x_{n}) \\
& =\sum_{r=0}^{\infty} m_{r+n}^{\hat{S} \Pi V^{*}}\left(\sum_{i_{1}} w^{i_{1}} \otimes w_{i_{1}}, \cdots, \sum_{i_{r}} w^{i_{r}} \otimes w_{i_{r}} \mid x_{1}, \cdots, x_{n}\right) \\
& =\sum_{\substack{r \geqslant 0 \\
i_{1}, \cdots, i_{r}}} w^{i_{r}} \cdots w^{i_{1}} \otimes m_{r+n}\left(w_{i_{1}}, \cdots, w_{i_{r}} \mid x_{1}, \cdots, x_{n}\right),
\end{aligned}
$$

for $x_{1}, \cdots, x_{n} \in \Pi V$. This structure is interpreted as a MC-element $\tilde{\xi}=\left(\tilde{\xi}_{n}\right)$ in the dgla

$$
\hat{S} \Pi V^{*} \otimes \overline{\operatorname{Hoch}}(V, V)=\bigoplus_{n \geqslant 1} \hat{S} \Pi V^{*} \otimes \operatorname{Hom}\left(T^{n} \Pi V, \Pi V\right)
$$

where

$$
\tilde{\xi}_{n}\left(x_{1}, \cdots, x_{n}\right)=\sum_{\substack{r \geqslant 1 \\ i_{1}, \cdots, i_{r}}} w^{i_{r}} \cdots w^{i_{1}} \otimes m_{r+n}\left(w_{i_{1}}, \cdots, w_{i_{r}} \mid x_{1}, \cdots, x_{n}\right) .
$$

(Note that the only the $r>0$ terms of the sum (12) contribute here.) Finally, interpreting the MC-element $\tilde{\xi} \in \hat{S} \Pi V^{*} \otimes \overline{\operatorname{Hoch}}(V, V)$ as an $L_{\infty}$-map $\mathbf{f}=\left(f_{r}\right)$ from $V$ into

$$
\overline{\operatorname{Hoch}}(V, V)=\bigoplus_{n \geqslant 1} \operatorname{Hom}\left(T^{n} \Pi V, \Pi V\right)
$$

we obtain the desired formula (10).

Now assume that $V$ is a cyclic $A_{\infty}$-algebra. Then the $L_{\infty}$-map $V \rightarrow \overline{\operatorname{Hoch}(V, V)}$ above factors through $\overline{\mathrm{CHoch}}(V, V)$, since a Maurer-Cartan twisting of a cyclic $A_{\infty^{-}}$ algebra is again cyclic. 


\subsection{Application to graph homology}

We now outline one application of the constructions in this section to graph homology. Let $(V, m)$ be an $L_{\infty}$-algebra which we assume to be finite-dimensional for simplicity and consider, as in Section 2.1 , the graded Lie algebra $\mathfrak{g}=\operatorname{Der}_{0}\left(\hat{S} \Pi V^{*}\right)$ consisting of (continuous) derivations of $\hat{S} \Pi V^{*}$ vanishing at zero. Note that $\mathfrak{g}$ has the same underlying space as $\overline{\mathrm{CE}}(V, V)$ but vanishing differential; particularly it does not depend on the $L_{\infty}$-structure on $V$. Consider the universal twisting of $V$; the $L_{\infty}$-structure on $\hat{S} \Pi V^{*} \otimes V$ corresponding to the twisting by the canonical element. This $L_{\infty}$-structure can itself be viewed as an element in $\mathrm{MC}\left(\mathfrak{g},\left(\hat{S} \Pi V^{*}, m\right)\right)$. Note, however, that this element does not lie in $\left(\hat{S} \Pi V^{*}\right)_{+} \otimes \Pi \mathfrak{g}$ and thus, we are in the framework of Remark 2.3.

It follows that there is a map from the CE complex of $\mathfrak{g}$ to that of $V$. At this stage we pass to the dual homological complexes $\mathrm{CE}_{*}(\mathfrak{g})$ and $\mathrm{CE}_{*}(V)$; thus $\mathrm{CE}_{*}(V) \cong$ $S \Pi V$ with the differential dual to $[-, m]$ and $\mathrm{CE}_{*}(\mathfrak{g})$ is the standard CE homological complex of the graded Lie algebra $\mathfrak{g}$. The switch to the dual language is not necessary, strictly speaking, but it is more natural for the interpretation of the result in terms of graph homology.

Note that both $\mathrm{CE}_{*}(V)$ and $\mathrm{CE}_{*}(\mathfrak{g})$ are $\mathrm{dg}$ coalgebras. It follows that there is a map of dg coalgebras: $\chi: \mathrm{CE}_{*}(V) \rightarrow \mathrm{CE}_{*}(\mathfrak{g})$. Since the coalgebra $\mathrm{CE}_{*}(\mathfrak{g})$ is cogenerated by the component $\Pi \mathfrak{g}$ it follows that such a map is determined by the composition $\mathrm{CE}_{*}(V) \rightarrow \mathrm{CE}(\mathfrak{g}) \rightarrow \Pi \mathfrak{g}$; abusing the notation we will denote the latter map also by $\chi$. Furthermore, $\chi$ can be viewed as a collection $\chi=\left(\chi_{1} \cdots, \chi_{r} \cdots\right)$ where $\chi_{r}:(\Pi V)^{\otimes r} \rightarrow \Pi \mathfrak{g}$. Moreover, since $\mathfrak{g}$ could be identified with $\prod_{n=1}^{\infty} \operatorname{Hom}\left(S^{n} V, V\right)$ the element $\chi_{r}\left(v_{1}, \cdots, v_{r}\right)$ is determined by its value on every collection $x_{1}, \cdots x_{n} \in \Pi V$ for $n=0,1, \cdots$.

Clearly, the same arguments also work in the $A_{\infty}$ situation, and also in the cyclic versions of both. We have the following result whose proof is essentially the same as that of Theorem 3.1:

\section{Proposition 3.3.}

1. Let $V$ be a finite-dimensional graded vector space:

(a) Let $\mathfrak{g}=\operatorname{Der}_{0} \hat{S} \Pi^{*} V$ be the graded Lie algebra of formal vector fields on $V$ with vanishing constant term and $m \in \mathrm{MC}(\mathfrak{g}, \mathbf{k})$ be an $M C$-element in $\mathfrak{g}$ giving $V$ the structure of an $L_{\infty}$-algebra. Then there is a map of $d g$ coalgebras $\chi: \mathrm{CE}_{*}(V) \rightarrow \mathrm{CE}_{*}(\mathfrak{g})$ determined by maps $\chi_{r}:(\Pi V)^{\otimes r} \rightarrow \Pi \mathfrak{g}, r=1,2, \ldots$, such that

$$
\chi_{r}\left(w_{1}, \cdots, w_{r}\right)\left(x_{1}, \cdots x_{n}\right)=m_{n+r}\left(w_{1}, \cdots, w_{r}, x_{1}, \cdots, x_{n}\right),
$$

where $w_{i}, x_{i} \in \Pi V ; r \geqslant 0$ and $n \geqslant 1$.

(b) Let $\mathfrak{h}=\operatorname{Der}_{0} \hat{T} \Pi^{*} V$ be the graded Lie algebra of formal noncommutative vector fields on $V$ with vanishing constant term and $m \in \mathrm{MC}(\mathfrak{h}, \mathbf{k})$ be an $M C$-element in $\mathfrak{h}$ giving $V$ the structure of an $A_{\infty}$-algebra. Then there is a map of dg coalgebras $\chi: \mathrm{CE}_{*}(V) \rightarrow \mathrm{CE}_{*}(\mathfrak{h})$ determined by a collection of maps $\chi_{r}:(\Pi V)^{\otimes r} \rightarrow \Pi \mathfrak{h}, r=1,2, \cdots$ such that

$$
\chi_{r}\left(w_{1}, \cdots, w_{r}\right)\left(x_{1}, \cdots x_{n}\right)=\sum_{\sigma \in S_{r}} \frac{1}{r !} m_{n+r}\left(\sigma\left(w_{1} \otimes \cdots \otimes w_{r}\right) \mid x_{1}, \cdots, x_{n}\right),
$$


where $w_{i}, x_{i} \in \Pi V ; r \geqslant 0$ and $n \geqslant 1$.

2. Let $V$ be a finite-dimensional graded vector space with an inner product (and thus, $\Pi V$ is a symplectic vector space):

(a) Let $\tilde{\mathfrak{g}}=\operatorname{Der}_{0} \hat{S} \Pi^{*} V$ be the graded Lie algebra of formal symplectic vector fields on $V$ with vanishing constant term and $m \in \mathrm{MC}(\tilde{\mathfrak{g}}, \mathbf{k})$ be an $M C$ element in $\tilde{\mathfrak{g}}$ giving $V$ the structure of a cyclic $L_{\infty}$-algebra. Then there is a map of dg coalgebras $\chi: \mathrm{CE}_{*}(V) \rightarrow \mathrm{CE}_{*}(\tilde{\mathfrak{g}})$ determined by a collection of maps $\chi_{r}:(\Pi V)^{\otimes r} \rightarrow \Pi \tilde{\mathfrak{g}}, r=1,2, \cdots$ such that

$$
\chi_{r}\left(w_{1}, \cdots, w_{r}\right)\left(x_{1}, \cdots x_{n}\right)=m_{n+r}\left(w_{1}, \cdots, w_{r}, x_{1}, \cdots, x_{n}\right),
$$

where $w_{i}, x_{i} \in \Pi V ; r \geqslant 0$ and $n \geqslant 1$.

(b) Let $\tilde{\mathfrak{h}}=\operatorname{Der}_{0} \hat{T} \Pi^{*} V$ be the graded Lie algebra of formal noncommutative symplectic vector fields on $V$ with vanishing constant term and

$$
m \in \operatorname{MC}(\tilde{\mathfrak{h}}, \mathbf{k})
$$

be an $M C$-element in $\tilde{\mathfrak{h}}$ giving $M$ the structure of an $A_{\infty}$-algebra. Then there is a map of $d g$ coalgebras $\chi: \mathrm{CE}_{*}(V) \rightarrow \mathrm{CE}_{*}(\tilde{\mathfrak{h}})$ determined by a collection of maps $\chi_{r}:(\Pi V)^{\otimes r} \rightarrow \Pi \tilde{\mathfrak{h}}, r=1,2, \cdots$ such that

$$
\chi_{r}\left(w_{1}, \cdots, w_{r}\right)\left(x_{1}, \cdots x_{n}\right)=\sum_{\sigma \in S_{r}} \frac{1}{r !} m_{n+r}\left(\sigma\left(w_{1} \otimes \cdots \otimes w_{r}\right) \mid x_{1}, \cdots, x_{n}\right),
$$

where $w_{i}, x_{i} \in \Pi V ; r \geqslant 0$ and $n \geqslant 1$.

Remark 3.4. The statement of Proposition 3.3 appears almost tautologically identical to that of Theorem 3.1; however the essential difference between these results is that we allow the value $r=0$ in the formulas for $\chi$ (but not in the formulas for $\mathbf{f}(9$ ), (10) ). This has the effect that $\chi$ does not give an $L_{\infty}$-map from $V$ into $\mathfrak{g}$. In fact, $\chi$ could be thought of as a curved $L_{\infty}$-map, cf. the last section concerning curved $L_{\infty}$-algebras. However, having such a map is sufficient to get an induced map on the CE homology.

The above result is particularly interesting when $V$ is a cyclic $L_{\infty^{-}}$or $A_{\infty}$-algebra. In that case letting the dimension of $V$ go to infinity we obtain the stable Lie algebra $\mathfrak{g}_{\infty}$ whose homology $\mathrm{CE}\left(\mathfrak{g}_{\infty}\right)$ is essentially Kontsevich's graph complex in the $L_{\infty^{-}}$ case and the complex of ribbon graphs in the $A_{\infty}$-case, $[\mathbf{9}, \mathbf{5}, \mathbf{6}]$. Therefore, we obtain a map from $\mathrm{CE}_{*}(V)$ to the appropriate version of the graph complex. This map is related to, but quite different from another construction, the so-called direct construction of Kontsevich which associates a graph homology class to $V$ itself. The precise connection between these constructions is the subject of a forthcoming paper.

\section{Homotopy fiber sequences of $L_{\infty}$-algebras}

In the previous section we constructed an $L_{\infty}$-map $\mathbf{f}: V \rightarrow \overline{\mathrm{CE}}(V, V)$ for an $L_{\infty^{-}}$ algebra $V$ which fits into a homotopy fiber sequence of $\mathrm{dg}$ vector spaces

$$
V \stackrel{\mathbf{f}}{\longrightarrow} \overline{\mathrm{CE}}(V, V) \longrightarrow \mathrm{CE}(V, V),
$$


and similarly in the $A_{\infty}$-algebra case. It is natural to ask whether these are actually homotopy fiber sequences in the category of $L_{\infty}$-algebras. In this section we show that this is indeed the case and explain the relation to work of Voronov and of FiorenzaManetti.

We begin by recalling the relevant notions. Note first that for any $L_{\infty}$-algebra $(V, m)$ there is defined another such $V[z, d z]:=V \otimes \mathbf{k}[z, d z]$ where $\mathbf{k}[z, d z]$ is the polynomial de Rham algebra on the interval; the evaluation maps $\left.\right|_{z=a}: \mathbf{k}[z, d z] \rightarrow$ $\mathbf{k}, a=0,1$, induce the corresponding evaluation maps $\left.\right|_{z=a}: V[z, d z] \rightarrow V$.

Definition 4.1. Let $V, W$ be two $L_{\infty}$-algebras and $f, g: V \rightarrow W$ be two maps between them. A homotopy between $f$ and $g$ is an $L_{\infty}$-algebra map $s: V \rightarrow W[z, d z]$ such that $\left.s\right|_{z=0}=f$ and $\left.s\right|_{z=1}=g$.

Note that a map $V \rightarrow W$ is by definition a (formal) cdga map $\hat{S} \Pi W^{*} \rightarrow \hat{S} \Pi V^{*}$; the latter is equivalent to an element in $\operatorname{MC}\left(W, \hat{S} \Pi V^{*}\right)$. We shall be particularly interested in the situation when $W$ is a dgla in which case $\left(\hat{S} \Pi V^{*}\right)_{+} \otimes W$ is a (formal) dgla. Then there is the following characterization of homotopy:

Proposition 4.2. Let $V$ be an $L_{\infty}$-algebra, $W$ be a dgla and $f, g \in \operatorname{MC}\left(W, \hat{S} \Pi V^{*}\right)$ be two corresponding $L_{\infty}$-maps. Then $f$ and $g$ are homotopic if and only if there exists an element $\xi \in\left(\hat{S} \Pi V^{*}\right)_{+} \otimes \Pi W$ such that $f=e^{\xi} g e^{-\xi}$.

Proof. This follows from a well-known characterization of homotopy in formal (or pronilpotent) dglas, cf. for example [1] , Theorem 4.4.

Furthermore we could form the homotopy category of dglas by formally inverting quasi-isomorphisms. There is also the notion of a homotopy fiber sequence.

Definition 4.3. For a dgla map $f: \mathfrak{g} \rightarrow \mathfrak{h}$ the homotopy fiber of $f$ is defined as $H_{f}:=$ $\left\{(g, h) \in \mathfrak{g} \oplus \mathfrak{h}[z, d z]:\left.h\right|_{z=0}=0,\left.h\right|_{z=1}=f(g)\right\}$; the projection onto $\mathfrak{g}$ is a dgla map $H_{f} \rightarrow \mathfrak{g}$. The sequence $H_{f} \rightarrow \mathfrak{g} \rightarrow \mathfrak{h}$ as well as any 3 -term sequence of dg Lie algebras homotopy equivalent to it will be called a homotopy fiber sequence of dglas.

For a homotopy fiber sequence $H_{f} \longrightarrow \mathfrak{g} \stackrel{f}{\longrightarrow} \mathfrak{h}$ the composite map $H_{f} \rightarrow \mathfrak{h}$ is homotopic to zero; moreover it possesses a semi-universal property: any map $\phi: \mathfrak{a} \rightarrow \mathfrak{h}$ of dglas which is homotopic to zero after composing with $f$ factors through $H_{f}$. Namely, if $s: \mathfrak{a} \rightarrow \mathfrak{h}[z, d z]$ is a nullhomotopy of $f \circ \phi$, then the factorization is given by $(\phi, s): \mathfrak{a} \rightarrow H_{f} \subset \mathfrak{g} \oplus \mathfrak{h}[z, d z]$. We will need a slight extension of this property: suppose that $\mathfrak{a}$ is an $L_{\infty}$-algebra and $\phi$ is an $L_{\infty}$-map from $\mathfrak{a}$ into $\mathfrak{g}$ whose composition with $f$ is nullhomotopic. Then the same formula as above gives an $L_{\infty}$-map from $\mathfrak{a}$ into $H_{f}$.

The dgla homotopy fiber $H_{f}$ should be compared with the dg homotopy fiber $C_{f}=\mathfrak{g} \oplus \Pi \mathfrak{h}$ with differential $d$ defined as follows. For $(g, h) \in \mathfrak{g} \oplus \Pi \mathfrak{h}$, set $d(g, h)=$ $(\partial g,-\partial h+f(g))$.

The following result is standard (see, e.g., [2, Lemma 3.2]). 
Proposition 4.4. The dg vector space $C_{f}$ is a retract of $H_{f}$, via maps $i: C_{f} \rightarrow H_{f}$ and $\pi: H_{f} \rightarrow C_{f}$ given by

$$
\begin{gathered}
i(g, h)=(g, f(g) z+h d z) ; \\
\pi(g, h(z, d z))=\left(g, \int_{0}^{1} h(z, d z)\right) .
\end{gathered}
$$

Remark 4.5. Note that $\mathrm{dg}$ fiber $C_{f}$ does not itself support a dgla structure. However Fiorenza and Manetti [2] use the retraction in Proposition 4.4 to transport the dgla structure of $H_{f}$ to an $L_{\infty}$-algebra structure on $C_{f}$.

Note that the category of dglas is a subcategory of the category of $L_{\infty}$-algebras, and this inclusion induces an equivalence of homotopy categories obtained by inverting respectively strict or $L_{\infty}$-quasi-isomorphisms. We can now define a homotopy fiber sequence of $L_{\infty}$-algebras.

Definition 4.6. The sequence of $L_{\infty}$-algebras and maps

$$
\mathfrak{a} \rightarrow \mathfrak{g} \rightarrow \mathfrak{h}
$$

is called homotopy fiber if it is $L_{\infty}$-quasi-isomorphic to a homotopy fiber sequence of dglas.

It is clear that the 'homotopy fiber' of an $L_{\infty}$-map $f: \mathfrak{g} \rightarrow \mathfrak{h}$ (i.e., an $L_{\infty}$-algebra $\mathfrak{a}$ which can be included in a fiber sequence $\mathfrak{a} \longrightarrow \mathfrak{g} \stackrel{f}{\longrightarrow} \mathfrak{h}$ ) is determined uniquely up to a non-canonical isomorphism in the homotopy category and that it possesses the analogous semi-universal property as the homotopy fiber of a dgla map.

\section{Theorem 4.7.}

1. (a) Let $V$ be an $L_{\infty}$-algebra; then the sequence

$$
V \stackrel{\mathbf{f}}{\longrightarrow} \overline{\mathrm{CE}}(V, V) \stackrel{g}{\longrightarrow} \mathrm{CE}(V, V))
$$

is a fiber sequence of $L_{\infty}$-algebras.

(b) Let $V$ be a cyclic $L_{\infty}$-algebra; then the sequence

$$
V \stackrel{\mathbf{f}}{\longrightarrow} \overline{\mathrm{CE}}(V, V) \stackrel{g}{\longrightarrow} \mathrm{CE}(V, V))
$$

is a fiber sequence of $L_{\infty}$-algebras.

2. (a) Let $V$ be an $A_{\infty}$-algebra; then the sequence

$$
V \stackrel{\mathbf{f}}{\longrightarrow} \overline{\operatorname{Hoch}(V, V)} \stackrel{g}{\longrightarrow} \operatorname{Hoch}(V, V)
$$

is a fiber sequence of $L_{\infty}$-algebras.

(b) Let $V$ be a cyclic $A_{\infty}$-algebra; then the sequence

$$
V \stackrel{\mathbf{f}}{\longrightarrow} \overline{\mathrm{CE}}(V, V) \stackrel{g}{\longrightarrow} \mathrm{CE}(V, V)
$$

is a fiber sequence of $L_{\infty}$-algebras. 
Proof. We restrict ourselves with proving the statement about $L_{\infty}$-algebras; the other parts are proved similarly. Denote by $\alpha=g \circ \mathbf{f}: V \rightarrow \mathrm{CE}(V, V)$ the composite $L_{\infty^{-}}$ map. Let us first prove that $\alpha$ is homotopic to zero. To this end we view $\alpha$ as an MC element in the dgla $\hat{S} \Pi V^{*} \otimes \Pi \mathrm{CE}(V, V)$. The zero map is represented by the CE differential $1 \otimes[, m]$ in this dgla. Observe that $\alpha=e^{\xi} d e^{-\xi}$ for $\xi=\sum w^{i} \otimes w_{i}$ where $w_{i}$ and $w^{i}$ are dual bases in $\Pi V$ and $\Pi V^{*}$ respectively, and $w_{i}$ is viewed as a (constant) derivation of $\hat{S} \Pi V^{*}$. It follows from Proposition 4.2 that $\alpha$ is indeed homotopic to zero. The homotopy has the following explicit form: $\alpha_{z}=e^{z \xi} d e^{-z \xi}+\xi d z$.

The arguments similar to those used to derive formulas (9) translate this homotopy to an $L_{\infty}$-map $s=\left\{s_{r}\right\}$ from $V$ to $\operatorname{CE}^{*}(V, V)[z, d z]$ :

$$
s_{r}\left(w_{1}, \cdots, w_{r}\right)=\left\{\begin{array}{l}
z^{r} f_{r}\left(w_{1}, \cdots, w_{r}\right), \text { if } r>1 \\
z f_{1}(w)+\hat{w} d z, \text { if } r=1 .
\end{array}\right.
$$

Here $\hat{w}$ refers to the constant derivation of $\hat{S} \Pi V^{*}$ associated to $w$ viewed as an element in $\Pi \mathrm{CE}(V, V)$.

The $L_{\infty}$-map $s$ gives rise to an $L_{\infty}$-map $\tilde{s}=\left\{\tilde{s}_{r}\right\}$ from $V$ to $H_{g} \subset \overline{\mathrm{CE}}^{*}(V, V) \oplus$ $\Pi \mathrm{CE}^{*}(V, V)$. Here $\tilde{s}_{r}:(\Pi V)^{\otimes n} \rightarrow \Pi H_{g}:$

$$
\tilde{s}_{r}\left(w_{1}, \cdots, w_{r}\right)=\left(f_{r}\left(w_{1}, \cdots, w_{r}\right), s_{r}\left(w_{1}, \cdots, w_{r}\right)\right) .
$$

It remains to show that $\tilde{s}_{1}: \Pi V \rightarrow \Pi H_{g}$ is a quasi-isomorphism. For this, it suffices to prove that the composite map $\pi \circ \tilde{s}_{1}: \Pi V \rightarrow \Pi C_{g}$ is a quasi-isomorphism. We have

$$
\left(\pi \circ \tilde{s}_{1}\right)(w)=\left(f_{1}(w), \int_{0}^{1} s_{1}(w)\right)=\left(f_{1}(w), \hat{w}\right) .
$$

Observe that $\mathrm{CE}(V, V)$ is the dg mapping cone of $f_{1}: V \rightarrow \overline{\mathrm{CE}}(V, V)$, and $g$ is the canonical map of $\overline{\mathrm{CE}}(V, V)$ into that cone. So we conclude the argument by appealing to the following general fact.

Lemma 4.8. Let

$$
X \stackrel{f}{\longrightarrow} Y \stackrel{g}{\longrightarrow} \operatorname{cone}(f)
$$

be a standard cofiber sequence of $d g$ vector spaces and let $C_{g}$ be the homotopy fiber of $g$; recall that $C_{g}$ has $Y \oplus \Pi Y \oplus X$ as its underlying graded vector space. Then the map $h: X \rightarrow C_{g}$ defined by $h(x)=(f(x), 0, x)$ is a quasi-isomorphism.

We now describe the relationship of Theorem 4.7 to Voronov's work on higher derived brackets $[\mathbf{1 4}, \mathbf{1 5}]$. We are indebted to the referee for bringing the precise connection to our attention. Voronov's starting point is an inclusion $K \hookrightarrow L$ of dgla's with abelian complement $W$. He shows that $L \oplus \Pi W$ may be endowed with an $L_{\infty^{-}}$ algebra structure so that the projection map $L \oplus \Pi W$ is a replacement of the original inclusion $K \hookrightarrow L$ by a fibration. This implies that $\Pi W$, equipped with Voronov's higher derived brackets, is a homotopy fiber of $K \hookrightarrow L$.

The inclusion $\overline{\mathrm{CE}}(V, V) \hookrightarrow \mathrm{CE}(V, V)$ (and its variations) we have considered in this section is a particular case of Voronov's setup, with complementary space $W=\Pi V$. His theory, applied in this case, shows that the $L_{\infty}$-algebra $(V, m)$ is a homotopy fiber of the inclusion. The new statement in Theorem 4.7 is the assertion that one obtains 
a homotopy fiber sequence $V \rightarrow \overline{\mathrm{CE}}(V, V) \rightarrow \mathrm{CE}(V, V)$, in which the first map is the map $f$ explicitly described in formulas (9) and (10).

Our explicit realisation of a homotopy fiber sequence may be compared with the main result of Fiorenza and Manetti [2]. Given an arbitrary dgla map $K \rightarrow L$, they construct a homotopy fiber sequence $C \rightarrow K \rightarrow L$ of $L_{\infty}$-algebras, where the underlying $\mathrm{dg}$ vector space of the homotopy fiber $L_{\infty}$-algebra $C$ is the homotopy fiber of $K \rightarrow L$ in the category of $d g$ vector spaces.

\subsection{Curved $L_{\infty}$-algebras and deformations}

We will now outline one application of the above theorem which, roughly, could be formulated as saying that an $L_{\infty}$-algebra $V$ controls the deformations of $V$ as an $L_{\infty^{-}}$ algebra which are trivial as deformations of curved $L_{\infty}$-algebras. A similar statement can, of course, be made in the $A_{\infty}$ or cyclic contexts; we will restrict ourselves to treating the $L_{\infty}$ case only.

Definition 4.9. A curved $L_{\infty}$-algebra structure on a graded vector space $V$ is a (continuous) odd derivation $m$ of the algebra $\hat{S} \Pi V^{*}$ such that $[m, m]=0$.

Note that such a derivation can be written as $m=m_{0}+m_{1}+\cdots$ where $m_{i}$ is an odd map $\hat{S}\left(\Pi V^{*}\right) \rightarrow \Pi V^{*}$ which is equivalent to a collection of $S_{n}$-invariant maps $(\Pi V)^{\otimes n} \rightarrow \Pi V$ subject to some constraints coming from the identity $[m, m]=0$. The difference with the notion of an $L_{\infty}$-algebra is that $m$ is not required to have zero constant term; consequently $m_{0}$ could be non-zero and $m_{1}$ does not necessarily square to zero.

For a curved $L_{\infty}$-algebra $(V, m)$ we could form its Chevalley-Eilenberg complex $\mathrm{CE}(V, V)$; its underlying space is Der $\hat{S}(V)$ and the differential is defined as the commutator with $m$, just as in the uncurved case. The condition $[m, m]=0$ ensures that the differential squares to zero, however there is no analogue of $\overline{\mathrm{CE}}(V, V)$ since $[, m]$ does not restrict to the subspace of derivations with the vanishing constant term. Clearly, $\operatorname{CE}(V, V)$ is a dgla. This dgla controls deformations of $V$; let us briefly recall the general setup of (derived) deformation theory, cf. for example [11].

Let $\mathfrak{g}$ be a dgla (or, more generally, an $L_{\infty}$-algebra). Let $\operatorname{Def}_{\mathfrak{g}}$ be the functor associating to a formal cdga $A$ the pointed set $\mathscr{M} \mathscr{C}(\mathfrak{g}, A)$ of MC-elements in $A_{+} \otimes \mathfrak{g}$ modulo gauge equivalence. Here we say that two elements $x_{0}, x_{1} \in \mathrm{MC}(\mathfrak{g}, A)$ are gauge equivalent if they are homotopic, i.e., there exists an MC element $X \in \mathrm{MC}(\mathfrak{g}[z, d z], A)$ such that $\left.X\right|_{z=0}=x_{0}$ and $\left.X\right|_{z=1}=x_{1}$. The base point in $\operatorname{Def}_{\mathfrak{g}}$ is the gauge class of the zero MC element. The functor $\operatorname{Def}_{\mathfrak{g}}$ is homotopy invariant in $\mathfrak{g}$, which means that a quasi-isomorphism $\mathfrak{g} \rightarrow \mathfrak{g}^{\prime}$ induces a natural bijection $\operatorname{Def}_{\mathfrak{g}}(A) \cong \operatorname{Def}_{\mathfrak{g}^{\prime}}(A)$.

Definition 4.10. A deformation of an $L_{\infty}$-algebra $V$ over a formal cdga $A$ is an MC-element in $A_{+} \otimes \overline{\mathrm{CE}}(V, V)$. Two such deformations are called equivalent if the corresponding MC-elements are gauge equivalent. Similarly a deformation of a curved $L_{\infty}$-algebra $V$ over a formal cdga $A$ is an MC-element in $A_{+} \otimes \mathrm{CE}(V, V)$. Two such deformations are called equivalent if the corresponding MC-elements are gauge equivalent.

Now let $\mathfrak{g} \rightarrow \mathfrak{h} \rightarrow \mathfrak{a}$ be a homotopy fiber sequence of $L_{\infty}$-algebras. Associated to it is the sequence of functors $\operatorname{Def}_{\mathfrak{g}} \rightarrow \operatorname{Def}_{\mathfrak{h}} \rightarrow \operatorname{Def}_{\mathfrak{a}}$ which is fiber in the sense that the 
preimage in $\operatorname{Def}_{\mathfrak{h}}$ of the base point in $\operatorname{Def}_{\mathfrak{a}}$ lies in the image of Def $f_{\mathfrak{g}}$. This could be easily seen by using the homotopy invariance of Def and reducing to a short exact sequence of dglas. Note that the map $\operatorname{Def}_{\mathfrak{g}} \rightarrow \operatorname{Def}_{\mathfrak{h}}$ is not necessarily injective; in fact it has an action of the group of homotopy self-equivalences of the functor $\operatorname{Def}_{\mathfrak{a}}$, however explaining this would take us too far afield and we refrain from giving further details.

We obtain the following result, which is an immediate consequence of theorem 4.7.

Corollary 4.11. A deformation of an $L_{\infty}$-algebra $V$ over a formal cdga $A$ which is trivial as its deformation as a curved $L_{\infty}$-algebra is always given by a twisting with some $M C$-element $\xi \in \mathrm{MC}(V, A)$.

In conclusion note that the constructions of this paper have an analogue in conventional homotopy theory. Let $X$ be a pointed space; denote by $\operatorname{Map}(X, X)$ and $\overline{\operatorname{Map}}(X, X)$ the space of all (respectively pointed) self-maps of $X$. There is an obvious fiber sequence of spaces

$$
\overline{\operatorname{Map}}(X, X) \rightarrow \operatorname{Map}(X, X) \rightarrow X
$$

which gives rise to the homotopy fiber sequence

$$
\Omega(X) \rightarrow \overline{\operatorname{Map}}(X, X) \rightarrow \operatorname{Map}(X, X),
$$

where $\Omega(X)$ is the space of based loops on $X$. This is parallel to any of the fiber sequences $(1),(2),(3)$ or (4). Note that there does not seem to be a simple description of the map $\Omega(X) \rightarrow \overline{\operatorname{Map}}(X, X)$.

\section{References}

[1] J. Chuang and A. Lazarev, Feynman diagrams and minimal models for operadic algebras, J. Lond. Math. Soc. 281 (2010), no. 2, 317-337.

[2] D. Fiorenza, M. Manetti, $L_{\infty}$ structures on mapping cones, Algebra Number Theory 1 (2007), 301-330.

[3] K. Fukaya, Deformation theory, Homological Algebra, and Mirror symmetry, Geometry and physics of branes (Como, 2001), 121-209, Ser. High Energy Phys. Cosmol. Gravit., IOP, Bristol, 2003.

[4] E. Getzler, Lie theory for nilpotent $L_{\infty}$ algebras, Ann. of Math. (2) 170 (2009), no. $1,271-301$.

[5] A. Hamilton, A super-analogue of Kontsevich's theorem on graph homology, Lett. Math. Phys. 76 (2006), no. 1. 37-55.

[6] A. Hamilton, A. Lazarev, Characteristic classes of $A_{\infty}$-algebras, J. Homotopy Relat. Struct. 3 (2008), no. 1, 65-111.

[7] A. Hamilton, Lazarev, Cohomology theories for homotopy algebras and noncommutative geometry, Alg. and Geom. Topology 9 (2009), 1503-1583.

[8] M. Kontsevich, Homological algebra of mirror symmetry, Proceedings of the International Congress of Mathematicians, Vol. 1, 2 (Zürich, 1994), 120-139, Birkhäuser, Basel, 1995. 
[9] M. Kontsevich, Formal Noncommutative Symplectic Geometry, The Gelfand Mathematical Seminars 1990-1992, 173-187, Birkhäuser Boston, Boston, MA, 1993.

[10] S. Merkulov, An $L_{\infty}$-algebra of an unobstructed deformation functor, Internat. Math. Res. Notices 2000, no. 3, 147-164.

[11] S. Merkulov, Frobenius $\infty_{\infty}$ invariants of homotopy Gerstenhaber algebras. I., Duke Math. J. 105 (2000), no. 3, 411-461.

[12] P. Nicolás, The bar derived category of a curved dg algebra, J. Pure Appl. Algebra 212 (2008), no. 12, 2633-2659.

[13] D. Quillen, Homotopical algebra, Springer LNM 43, 1967.

[14] T. Voronov, Higher derived brackets and homotopy algebras, J. Pure Appl. Algebra 202 (2005), no. 1-3, 133-153.

[15] T. Voronov, Higher derived brackets for arbitrary derivations, Travaux mathématiques. Fasc. XVI, 163-186, Trav. Math., XVI, Luxembourg, 2005.

Joseph Chuang J.Chuang@city.ac.uk

Centre for Mathematical Science, City University London, London, EC1V 0HB, UK

Andrey Lazarev al179@leicester.ac.uk

University of Leicester, Department of Mathematics, Leicester, LE1 7RH, UK 